\title{
ACUPUNTURA E AURICULOTERAPIA COMO MÉTODOS NÃO FARMACOLÓGICOS DE ALÍVIO DA DOR NO PROCESSO DE PARTURIÇÃO
}

\author{
Fabiane Cherobin ${ }^{1}$, Arnildes Rodrigues Oliveira ${ }^{2}$, Ana Maria Brisola ${ }^{3}$
}

\begin{abstract}
RESUMO: O processo de parturição correlaciona as alterações fisiológicas a sentimentos e a valores socioculturais. A dor é um dos importantes sinais nesse processo. Porém, se não controlada, pode causar inúmeros efeitos colaterais indesejados. Para minimizá-los, métodos não farmacológicos podem atuar no alívio da dor. Assim, o objetivo desta pesquisa foi analisar os resultados da acupuntura e auriculoterapia como controle da dor, por meio de pesquisa convergente assistencial, entre junho e setembro de 2015. Concordaram em participar do estudo 19 parturientes admitidas em trabalho de parto no centro obstétrico de uma maternidade pública de Santa Catarina. Os resultados demonstraram que $n=15(79 \%)$ das mulheres obtiveram alívio da dor nos primeiros 30 minutos de tratamento. Os resultados desta pesquisa trazem animadoras perspectivas para a assistência ao trabalho de parto por se tratarem de métodos de baixo custo e seguros, aumentando o número de alternativas não farmacológicas para as parturientes.
\end{abstract}

DESCRITORES: Acupuntura; Auriculoterapia; Analgesia; Trabalho de parto; Dor.

\section{ACUPUNCTURE AND AURICULOTHERAPY AS NON-PHARMACOLOGICAL PAIN RELIEF METHODS IN THE CHILDBIRTH PROCESS}

ABSTRACT: The childbirth process correlates the physiological changes with feelings and socio-cultural values. Pain is one of the important signals in this process. However, if uncontrolled, can cause many unwanted side effects. To minimize them, nonpharmacological methods can act to relieve pain. The aim of this study was to analyze the results of acupuncture and auriculotherapy as pain control, through a convergent care study, performed between June and September 2015. A total of 19 pregnant women in labor admitted to the obstetrics center of a public hospital of Santa Catarina agreed to participate in the study. Results showed 15 $(79 \%)$ women feltpain relief in the first 30 minutes of the treatment. These results bring exciting prospects for labor care because the methods are inexpensive and safe, increasing the number of non-pharmacological alternatives for women in labor.

DESCRIPTORS: Acupuncture; Auriculotherapy; Analgesia; Labor; Pain.

\section{ACUPUNTURA Y AURICULOTERAPIA COMO MÉTODOS NO FARMACOLÓGICOS DE ALIVIO DEL DOLOR EN EL PROCESO DE PARTO}

RESUMEN: El proceso del parto asocia las alteraciones fisiológicas a sentimientos y a valores socioculturales. El dolor es un de los señales importantes en ese proceso. Sin embargo, si no es controlada, puede traer innúmeros efectos colaterales. Para minimizarlos, métodos no farmacológicos pueden actuar en el alivio del dolor. Así, fue objetivo de esta investigación analizar los resultados de la acupuntura y auriculoterapia como control del dolor, por medio de investigación convergente asistencial, entre junio y septiembre de 2015. Participaron del estudio 19 parturientes admitidas en trabajo de parto en el centro obstétrico de una maternidad pública de Santa Catarina. Los resultados muestran que $\mathrm{n}=15$ (79\%) de las mujeres obtuvieron alivio del dolor en los primeros 30 minutos de tratamiento. Los resultados de esta investigación revelan animadoras perspectivas para la asistencia al trabajo de parto por el pequeño costo y la seguridad de los métodos, aumentando el número de alternativas no farmacológicas para las parturientes.

DESCRIPTORES: Acupuntura; Auriculoterapia; Analgesia; Trabajo de parto; Dolor.

${ }^{1}$ Enfermeira. Especialista em Acupuntura. Enfermeira Residente Multiprofissional em Saúde Materno Infantil. Maternidade Darcy Vargas. Joinville, SC, Brasil.

${ }^{2}$ Enfermeira. Enfermeira Obstetra. Mestre em Gestão do cuidado. Docente de Enfermagem. Faculdade de Enfermagem da Associação Educacional Luterana Bom Jesus. Joinville, SC, Brasil.

${ }^{3}$ Enfermeira. Enfermeira Obstetra. Especialista em Acupuntura. Maternidade Darcy Vargas. Joinville, SC, Brasil.

Autor Correspondente:

Fabiane Cherobin

Associação Educacional Bom Jesus

R. Princesa Isabel, 438 - 89201-270 - Joinville, SC, Brasil

E-mail: fabicherobin@ibest.com.br
Recebido: $12 / 02 / 2016$

Finalizado: 16/08/2016 


\section{○ INTRODUÇÃO}

O trabalho de parto é um conjunto de alterações fisiológicas (contrações uterinas de intensidade e frequência crescente que resultam na dilatação progressiva do colo uterino e descida da apresentação fetal) que ocorrem dentro de um período de tempo e tem como objetivo o nascimento do feto ${ }^{(1)}$. Porém, o processo de parturição e parto vai muito além do aspecto fisiológico. Tem significado único e diferenciado para a vida da mulher e da sua família, envolvendo sentimentos de receios, angústias e valores socioculturais ${ }^{(2)}$. Dentre os medos associados ao parto, encontra-se a dor.

A dor representa um importante sinal no trabalho de parto ${ }^{(3-4)}$ e tem sido considerada como quinto sinal vital ${ }^{(5)}$. O componente mais importante da dor é a dilatação do colo uterino somada a outros fatores como contração e distensão das fibras uterinas, relaxamento do canal de parto, tração de anexos e peritônio, pressão na uretra, bexiga e outras estruturas pélvicas e, ainda, pressão sobre as raízes do plexo lombo sacro ${ }^{(4,6)}$.

O descontrole da dor obstétrica produz uma série de alterações na fisiologia materna, as quais somadas àquelas que a própria gestação causa, podem resultar em efeitos colaterais indesejáveis no feto e na mãe, entre os quais destacamos a hiperventilação, o aumento do consumo de oxigênio, o aumento das concentrações plasmáticas de betaendorfinas e catecolaminas que diminuem o fluxo sanguíneo placentário, bem como o aumento de renina (estimulante da produção de angiotensina I e II) e das concentrações de ácidos graxos livres, entre outras alterações ${ }^{(5)}$.

Diante deste quadro, a restrição do desconforto da parturiente é de extrema importância. A percepção dos estímulos dolorosos pode ser reduzida por meio de medidas classificadas como farmacológicas e não farmacológicas. Estas últimas promovem sensação de bem-estar na mulher e diminuem o estresse no parto, reduzindo o uso de medidas alopáticas ${ }^{(7)}$.

Dentre os métodos não farmacológicos podemos citar a acupuntura, auriculoterapia, fitoterapia, aromaterapia, moxabustão, massagem, banho terapêutico, presença de doula e acompanhante, e bola suíça.

Neste trabalho o enfoque será dado para os dois primeiros métodos descritos acima, contemplados na Medicina Tradicional Chinesa (MTC).

A MTC é praticada há cerca de 5.000 anos no Oriente e atualmente é utilizada em diversos países ocidentais como forma de ampliar as possibilidades de condutas terapêuticas. Trata-se de uma contribuição extremamente significativa para a melhoria das condições de vida da população ${ }^{(8)}$.

No Brasil, a prática das técnicas chinesas iniciou-se timidamente no século XIX, mas só foi incorporada nas instituições no século XX, a partir da década de 80. Posteriormente, o Ministério da Saúde criou a Política Nacional de Práticas Integrativas e Complementares em Saúde visando à integralidade da atenção à saúde ${ }^{(9)}$.

Dentre as diversas técnicas da MTC, encontra-se a acupuntura, termo derivado do latim: agulha (acus) e puntura (puntura). A terapia considera que em nosso corpo existem aproximadamente 360 acupontos de meridianos e a maioria deles está associado com estruturas do sistema nervoso periférico, feixes periféricos e vasos sanguíneos. A inserção da agulha ativa os receptores dos dendritos dos neurônios sensoriais na pele, músculos e tecidos moles. Essa estimulação gera sinais elétricos, conhecidos como potencial de ação, que percorrem os dendritos, chegam ao corpo celular, posteriormente ao axônio e finalmente ao cérebro por meio das sinapses ${ }^{(10)}$.

Após os impulsos de dor chegarem ao córtex cerebral, endorfinas, serotonina, hormônio adrenocorticotrófico (ACTH) e ácido gama-aminobutírico (GABA) são liberados em diferentes locais para modular os sinais da dor ${ }^{(10)}$.

Outra técnica milenar é a acupuntura auricular ou auriculoterapia, no latim: orelha (auris), pequena orelha (auricula) e do grego: terapia (terapien). É definida como uma terapia que provoca estímulos em pontos específicos da orelha externa, a qual representa um feto de cabeça para baixo e reflete todos os órgãos do corpo humano. Essa parte do corpo humano é extremamente inervada e possibilita, através de estimulação por agulhas, sementes ou outros materiais, a condução dos sinais elétricos, 
sensibilizando regiões do cérebro como o tronco cerebral, córtex e outras regiões do encéfalo. Cada ponto auricular está diretamente ligado a um ponto no encéfalo que se conecta ao órgão ou região do corpo $^{(11)}$.

O interesse pela acupuntura e auriculoterapia na analgesia durante o trabalho de parto e parto se justifica pelas inúmeras vantagens que elas representam para o binômio mãe-filho: não alteram os níveis de consciência materna, proporcionando o seu envolvimento durante todo o processo de parto e após o parto, não impede o uso de outras formas de analgesias, é uma opção viável economicamente, e é uma técnica que é segura, já que não há registro de efeitos colaterais em sua aplicação.

Os objetivos desse trabalho foram: a análise dos resultados dos métodos não farmacológicos pesquisados como controle da dor no processo de parturição; elencar pontos de acupuntura e auriculoterapia para utilizar no tratamento; avaliar o grau da dor por meio da escala visual analógica durante o trabalho de parto e parto utilizando as terapias propostas, e conhecer a percepção da parturiente com relação aos métodos não farmacológicos utilizados.

\section{METODOLOGIA}

Foi realizada uma pesquisa convergente assistencial entre os meses de junho e setembro de 2015, envolvendo a participação consentida de 19 parturientes admitidas em trabalho de parto no centro obstétrico de uma maternidade pública no estado de Santa Catarina. A metodologia convergente assistencial consiste em uma modalidade de pesquisa qualitativa, caracterizada pela correlação entre pesquisa, assistência e participação dos sujeitos envolvidos na prática, juntamente ao processo de construção do conhecimento ${ }^{(12)}$.

Para a acupuntura, as agulhas utilizadas eram descartáveis de 0,25x30 mm e para a auriculoterapia utilizaram-se esferas de cristal polido com 1,5mm, aderidas à pele com fitas hipoalérgicas. Quando a parturiente solicitava o cuidado, iniciava-se o tratamento. Primeiramente a parturiente era posicionada em decúbito lateral ou sentada, realizada a antissepsia com álcool $70 \%$ nos locais onde seriam realizadas as punturas e a auriculoterapia. Foram utilizados os pontos Yintang (envolvido na diminuição da ansiedade e do medo); IG4 (analgésico); VC2 (envolvido no alívio da dor na sínfise púbica); BP6 (envolvido no alívio da dor de contração); B60 (envolvido no relaxamento dos tendões e músculos e no alívio da dor de contração); F3 (envolvido no relaxamento dos músculos e tendões) e na região dorsal B31, B32 (envolvidos no fortalecimento da região lombo-sacra) ${ }^{(13-15)}$. Na auriculoterapia utilizaram-se os pontos shenmen (analgésico), sistema nervoso central (analgésico) e rim (essa tríade é utilizada no início de qualquer tratamento de auriculoterapia); útero (dificuldade no trabalho de parto); endócrino (regula distúrbios da função endócrina); abdômen (contrações) e subcórtex (analgésico) ${ }^{(16-17)}$ conforme definição da acupuntura clássica chinesa.

Para definir a percepção da dor durante o trabalho de parto, foi utilizada a escala visual analógica (EVA) que varia de 0 a 10, em que o 0 é ausência de dor e 10 significa dor intensa ${ }^{(18)}$. O alívio da dor foi classificado inicialmente comparando a EVA inicial com o valor da EVA em intervalos de 30min.

Para as análises estatísticas dos dados, foram selecionadas como variáveis dependentes: a avaliação da intensidade da dor em intervalos de 30 minutos, os motivos de encerramento da técnica e a avaliação feita pela puérpera do alívio da dor proporcionado. Como variáveis de controle foram analisadas a idade, estado civil, nível de escolaridade, número de gestações anteriores, número de consultas de pré-natal, conhecimento prévio sobre as terapias complementares e classificação da intensidade da dor antes do início do tratamento.

Nas primeiras 24 horas após o nascimento do bebê, foi aplicada entrevista semiestruturada com objetivo de avaliar a eficácia dos tratamentos utilizados no alívio da dor durante o trabalho de parto. Os nomes próprios das parturientes foram substituídos por pseudônimos de flores para preservar o anonimato. As variáveis dependentes e de controle foram avaliadas no programa Microsoft Excel e submetidos à análise estatística simples e as perguntas abertas foram analisadas em três fases: leitura, agrupamento das informações por semelhança e a relação com o método de tratamento complementar.

As parturientes foram informadas sobre os objetivos e métodos do estudo e puderam optar ou não 
pela participação, sem qualquer prejuízo ao seu atendimento. Participaram mulheres primíparas e multíparas a termo; de risco habitual; maiores de 18 anos; residentes no município da pesquisa; e que não utilizaram analgesia previamente. O projeto desta pesquisa foi aprovado pelo Comitê de Ética em Pesquisa sob parecer número 970.689.

\section{RESULTADOS}

A idade média das voluntárias foi de 24,8 anos, variando de 18 a 35 anos, todas referindo ser casadas ou em união consensual. Com relação ao grau de instrução, sete (37\%) possuíam o ensino médio completo, cinco (26\%) ensino fundamental incompleto, três (16\%) ensino médio incompleto, duas $(11 \%)$ ensino fundamental completo, uma (5\%) cursava o ensino superior e uma (5\%) era graduada. Todas as parturientes realizaram pré-natal no Sistema Único de Saúde, sendo que 17 (89\%) tiveram seis ou mais consultas, conforme preconizado pelo Ministério da Saúde.

Analisando os dados obstétricos, 12 (63\%) tiveram gestações anteriores, destas, cinco (42\%) tinham histórico de parto normal, quatro (33\%) cesariana, duas (17\%) com abortos prévios e uma (8\%) com parto normal e cesariana.

Com relação às terapias complementares: acupuntura, auriculoterapia, moxabustão, fitoterapia, Do in, tuiná e ventosaterapia, 11 (58\%) tinham conhecimento de alguma das técnicas citadas, dentre essas, dez (91\%) citaram a acupuntura e uma (9\%) a auriculoterapia. Observou-se que uma (9\%) das parturientes já haviam feito tratamento com acupuntura, mas nenhuma havia utilizado os métodos durante a gestação.

Para o tratamento, o tempo mínimo estabelecido de puntura foi de 30 minutos. A maioria, 12 (63\%), permaneceu por $1 \mathrm{~h}$. Apenas sete $(36 \%)$ persistiram até $1 \mathrm{~h} 30 \mathrm{~min}$, duas $(10,5 \%)$ finalizaram o tratamento de acupuntura com $2 \mathrm{~h}$ e uma $(5,2 \%)$ das parturientes encerrou após 5h30min. Observou-se que 19 $(100 \%)$ das parturientes mantiveram a auriculoterapia após a retirada das agulhas.

No que se refere à interrupção da técnica, esta ocorreu por vários motivos, sendo o principal deles ( $\mathrm{n}=11$ (55\%) dos casos) o desejo da própria cliente (justificando a preferência pelo banho terapêutico, à deambulação ou pelo incômodo das agulhas). Em cinco (25\%) dos casos, a interrupção das técnicas ocorreu por solicitação de analgesia, três (15\%) encerraram o tratamento devido ao nascimento e um (5\%) foi devido ao término do plantão da enfermeira residente (neste caso específico, a parturiente permaneceu com auriculoterapia no contraturno e no início do plantão foi reiniciada a acupuntura).

Com relação à análise da redução da dor (Figura 1), observa-se que 15 (79\%) das mulheres tratadas obtiveram algum alívio nos 30 minutos iniciais. Na avaliação, após 1h, seis (46\%) permaneceram com o mesmo grau de dor e quatro (31\%) obtiveram um alívio maior. Após $1 \mathrm{~h} 30 \mathrm{~min}$ as parturientes referiram piora no sintoma quatro (57\%). Com $2 \mathrm{~h}$ de tempo decorrido, metade das pacientes apresentou piora e a outra metade manteve a mesma classificação da dor.

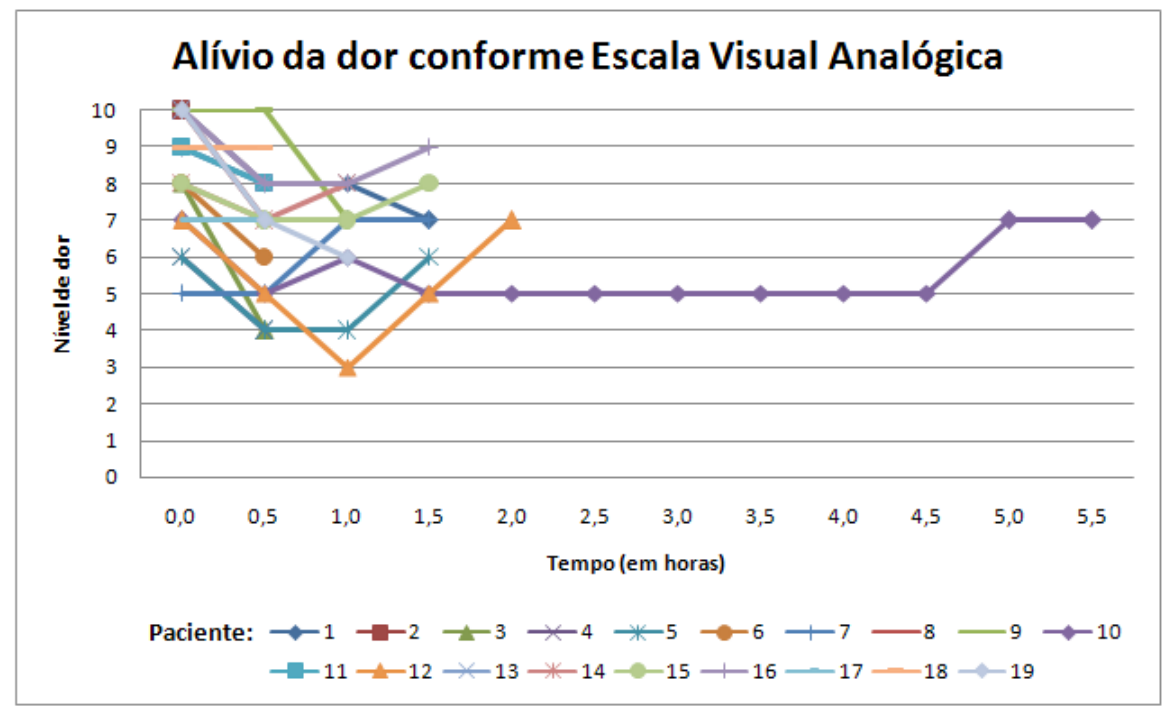

Figura 1 - Classificação da dor em intervalos de 30min conforme cada parturiente. Joinville, SC, Brasil, 2015 
No puerpério, quando questionadas sobre a eficácia do tratamento durante o trabalho de parto, 17 $(89,6 \%)$ entrevistadas responderam que houve alívio da dor, uma $(5,2 \%)$ referiu piora e uma $(5,2 \%)$ não soube responder.

Questionadas quanto ao fato de indicarem o método para outras mulheres, a resposta foi $100 \%$ positiva.

Ao responderem a última questão: "Por que recomendaria esse tipo de atendimento a outras pessoas?" diversas opiniões foram relatadas:

[...] valeu a pena, se não fosse pelas agulhas eu teria desistido antes [...]. (Violeta)

[...] gostei, melhor que analgesia. Aliviou até quase no final, mas a analgesia também não tira toda a dor [...]. (Begonia)

[...] além de ajudar nas dores, acalma, tranquiliza e diminui o cansaço [...]. (Hortência)

[...] gostei, senti melhora da dor pós-cesárea [...]. (Azaléia)

Nesse último depoimento a puérpera foi encaminhada para cesariana por intercorrências com o feto.

\section{DISCUSSÃO}

A taxa de primíparas que utilizaram métodos não farmacológicos foi semelhante ao encontrado em outro estudo: 32,9\%. Já em comparação ao nível de instrução houve diferenças: o estudo mostra que $31,7 \%$ das parturientes tinham idade entre 10 e 19 anos, e haviam estudado de 11 a 14 anos ${ }^{(19)}$.

O número de gestações prévias pode ter sido um fator importante na decisão de participar do tratamento com acupuntura e auriculoterapia, visto que nos trabalhos de parto anteriores as parturientes já haviam experimentado métodos não farmacológicos como bola suíça, banho terapêutico, deambulação, direito ao acompanhante, liberdade de posições durante o trabalho de parto e dieta líquida irrestrita.

Ao questionar as parturientes sobre o conhecimento acerca das terapias complementares, observou-se prevalência das mulheres que conheciam alguma das técnicas. Contrariando esses dados, uma pesquisa com 120 puérperas demonstrou que pequena parcela $(23,3 \%)$ possuía vaga noção sobre o assunto e quase a mesma proporção $(26,5 \%)$ possuía conhecimentos sobre as técnicas ${ }^{(20)}$.

Na presente pesquisa não foram diferenciadas as mulheres que só ouviram falar sobre as terapias daquelas que tinham entendimento dos métodos. O destaque neste tópico foi o fato de que, dentre os métodos conhecidos, o mais citado foi a acupuntura. Este conhecimento prévio sobre o assunto pode ter facilitado a adesão para o tratamento no trabalho de parto.

No entanto, pesquisa realizada em Fortaleza ${ }^{(21: 35)}$ destaca que "[...] fica evidente a descrição de todo o processo parturitivo baseado na sensação dolorosa, seja ela presente ou ausente, o que reafirma a dor como algo muito forte em nossa cultura $[\ldots . .]^{\prime \prime}$.

Com relação ao tempo de tratamento, um ensaio clínico ${ }^{(17)}$ mostrou que as parturientes foram mais persistentes no tempo de permanência com a acupuntura: $84 \%$ delas permanecendo por $1 \mathrm{~h}, 53 \%$ por $1 \mathrm{~h} 30 \mathrm{~min}$ e $43 \%$ por $2 \mathrm{~h}$. Porém, em auriculoterapia as parturientes permaneceram menos tempo, sendo $93 \%$ na primeira hora e chegando a $34 \%$ na segunda hora.

Em estudo dinamarquês(22) sinalizou-se que após $2 \mathrm{~h} 30 \mathrm{~min}$, metade das parturientes ainda utilizava a acupuntura. Esses dados mostram que as participantes da pesquisa atual tiveram pequena tolerância quando comparadas a estudos anteriores. Pode-se presumir que há influência cultural, baixa tolerância à dor, falta de preparação durante o pré-natal e algum fator relacionado ao nível de escolaridade.

O tempo reduzido observado no presente estudo pode ser justificado pela argumentação da preferência pelo banho terapêutico, deambulação ou a presença incômoda de agulhas. Evidenciou-se que $75,2 \%$ das mulheres em trabalho de parto optaram pelo banho morno e $85,1 \%$ pela deambulação ${ }^{(23)}$. 
Um ensaio clínico realizado com 120 parturientes mostrou que o alívio da dor alcançado nos primeiros 30 minutos foi de 3 a 4 vezes maior no grupo que recebeu tratamento comparado ao grupo controle. Essa diferença foi observada aos 60 minutos e 90 minutos $^{(17)}$.

Um estudo randomizado controlado(22) envolvendo 600 parturientes demonstrou que $59 \%$ tiveram alívio da dor. Vale acrescentar que a maioria dessas parturientes (53\%) demonstrou desejo em utilizar o método numa próxima gestação.

Na presente pesquisa, a maior parcela das mulheres obteve alívio da dor no início do tratamento, permanecendo ou piorando com o passar do tempo. Essa piora também pode estar relacionada com o aumento da dilatação e das contrações progressivas, próprias da evolução do parto. Trata-se apenas de hipótese, pois esses dados não foram pesquisados. Outra suposição poderia estar relacionada à baixa eficácia do tratamento não farmacológico utilizado, o que se opõe aos estudos citados acima.

Durante o feedback com as puérperas, observou-se a eficácia do tratamento, com todas respondendo positivamente. Corroborando com estes dados, um estudo transversal de abordagem qualitativa mostrou que uso dos métodos não farmacológicos foi fator positivo devido ao alívio da dor ou ausência de outros desconfortos ${ }^{(24)}$.

Diversas percepções vieram à tona quando as entrevistadas falaram sobre os métodos utilizados no trabalho de parto. Algumas compararam com os métodos farmacológicos, referindo que nem mesmo estes anulam a dor. Outras mencionaram a acupuntura propiciando um efeito encorajador neste processo. Dentre os pontos da MTC utilizados, estava o Yintang, que auxilia na redução do medo e ansiedade e sua eficácia foi relatada por uma das puérperas.

Nem todos os métodos são eficazes no alívio da dor, porém promovem redução do nível de estresse, medo, ansiedade e promovem a satisfação ${ }^{(24)}$. A dor do parto é fisiologicamente real, porém percebida de forma diferente por cada mulher. Independente do método utilizado para amenizar o sofrimento, deve-se pensar em seu objetivo que é o enfretamento da dor, tornando a passagem do trabalho de parto menos agressiva e dolorosa.

\section{- CONCLUSÃO}

A intensidade da dor é individual, entretanto, não é apenas ela que trará a percepção da experiência do parto como positiva ou negativa. Não se pode ter um olhar fragmentado sobre este processo considerando apenas o sofrimento físico. O trabalho de parto é um momento que reúne angústias, medos e ansiedade, podendo potencializar a dor.

Considerando os resultados dessa pesquisa, as perspectivas são positivas para a assistência ao trabalho de parto por se tratar de métodos de baixo custo e seguros, aumentando o número de alternativas não farmacológicas para as parturientes. Tais métodos podem funcionar como uma ação inicial ou combinada com outras técnicas, capazes de preservar a naturalidade do processo de parturição e tornando-o mais tranquilo.

Para o aprimoramento desses métodos são necessárias pesquisas nacionais futuras, com maior abrangência populacional, e que objetivam mostrar a eficiência das técnicas da Medicina Tradicional Chinesa, bem como de outros métodos que ainda possam surgir. Assim, cada vez mais, poderão ser ofertadas às parturientes maneiras que venham a contribuir para o alcance da serenidade durante $o$ trabalho de parto.

As limitações deste estudo estão relacionadas ao conhecimento restrito por parte das parturientes sobre a eficácia das técnicas abordadas, dificultando a amplitude do estudo.

\section{- REFERÊNCIAS}

1. Montenegro CAB, Rezende J. Obstetrícia. 11ª ed. Rio de Janeiro: Guanabara Koogan; 2011. Parto. p $244-62$.

2. Neumann ABT, Garcia CTF. A percepção da mulher acerca do acompanhante no processo de parturição. Rev. 
Contexto Saúde. [Internet] 2011; 10(20) [acesso em 10 out 2015]. Disponível:

https://www.revistas.unijui.edu.br/index.php/contextoesaude/article/view/1509.

3. Cunha AA. Analgesia e anestesia no trabalho de parto e parto. Rev. Femina. [Internet] 2010;38(11) [acesso em 03 fev 2016]. Disponível: http://www.febrasgo.org.br/site/wp-content/uploads/2013/05/feminav38n11_599-606.pdf.

4. Ministério da Saúde (BR). Secretaria de Políticas de Saúde. Área técnica de Saúde da Mulher. Parto, Aborto e Puerpério. $1^{\text {a }}$ ed. Brasília: Ministério da Saúde; 2001.

5. Martini JG, Becker SG. Acupuntura na analgesia do parto: percepções das parturientes. Esc. Anna Nery. [Internet] 2009; 13(3) [acesso em 08 set 2015]. Disponível: http://dx.doi.org/10.1590/S1414-81452009000300019.

6. Santana LS, Gallo RBS, Ferreira CHJ, Quintana SM, Marcolin AC. Localização da dor na fase ativa do trabalho de parto. Rev. dor. [Internet] 2013; 14(3) [acesso em 30 jan 2016]. Disponível: http://dx.doi.org/10.1590/S180600132013000300006.

7. Sartori AL, Vieira F, Almeida NAM, Bezerra ALQ, Martins CA. Estrategias no farmacológicas para aliviar el dolor durante el proceso del parto. Enfermeria Global. [Internet] 2011; (21) [acesso em 15 set 2015]. Disponível: http://revistas.um.es/eglobal/article/viewFile/116001/109961.

8. Secretaria Municipal de Saúde (SP). Caderno Temático de Medicina Tradicional Chinesa. $1^{\text {a }}$ edição. São Paulo: Secretaria Municipal de Saúde; 2003.

9. Ministério da Saúde (BR). Secretaria de Atenção à Saúde. Departamento de Atenção Básica. Política nacional de práticas integrativas e complementares no SUS: atitude de ampliação de acesso. $2^{a}$ ed. Brasília: Ministério da Saúde; 2015.

10. Ma Y, Ma M, Cho ZH. Acupuntura para controle da dor: um enfoque integrado. $1^{a}$ ed. São Paulo: Roca; 2006. Mecanismos periféricos da acupuntura. p.31-47

11. de Sousa EMD, da Trindade AKF, Pereira IC. Auriculoterapia: terapia milenar e eficiente no tratamento de enfermidades. Rev. Conceitos. [Internet] 2014; 20(1) [acesso em 02 dez 2015]. Disponível:

http://www.adufpb.org.br/site/wp-content/uploads/2014/09/REVISTA-CONCEITOS-20-2.pdf\#page=89.

12. Pivoto FL, Lunardi Filho WD, Santos SSC, Lunardi VL. Pesquisa convergente-assistencial: revisão integrativa de produções científicas da enfermagem. Rev. Texto Contexto Enferm. [Internet] 2013; 22(3) [acesso em 24 jan 2016]. Disponível: http://dx.doi.org/10.1590/S0104-07072013000300034.

13. Campligna H. Domínio do Yin- Da fertilidade à maternidade: a mulher e suas fases na medicina tradicional chinesa. $1^{a}$ ed. São Paulo: Roca; 2010. Parto. p. 111-20

14. Maciocia G. Os fundamentos da medicina chinesa. $2^{a}$ ed. São Paulo: Roca; 2007.

15. Serafi M, Lopes C. Ponto! Atlas topográfico de Acupuntura. 2ª ed. São Paulo: Ponto Crítico; 2008.

16. Souza MP. Tratado de auriculoterapia. $1^{\text {a }}$ ed. Brasília: Novo Horizonte; 2007.

17. Knobel R. Técnicas de acupuntura para alívio da dor no trabalho de parto, ensaio clínico. [tese]. Campinas: Universidade Estadual de Campinas (SP): Faculdade de Ciências Médicas; 2002.

18. Calik KY, Komurcu N. Effects of SP6 Acupuncture Point Stimulation on Labor Pain and Duration of Labor. Iran Red Crescent Med J. [Internet] 2014; 16(10) [acesso em 20 jan 2015]. Disponível:

http://dx.doi.org/10.5812/ircmj.16461.

19. Leal MC, Pereira APE, Domingues RMSM, Theme Filha MM, Dias MAB, Nakamura-Pereira M, et al. Intervenções Obstétricas durante o trabalho de parto e parto em mulheres brasileiras de risco habitual. Cad. Saúde Pública. [Internet] 2014; 30(Suppl.1) [acesso em 28 jan 2016]. Disponível: http://dx.doi.org/10.1590/0102-311X00151513.

20. de Almeida JM, Acosta LG, Pinhal MG. Conhecimento das puérperas com relação aos métodos não farmacológicos de alívio da dor do parto. Reme, Rev. Min. Enferm. [Internet] 2015; 19(3) [acesso em 04 fev 2016]. Disponível: http://dx.doi.org/10.5935/1415-2762.20150054. 
21. de Oliveira ASS, Rodrigues DP, Guedes MVC, Felipe GF. Percepção de mulheres sobre a vivência do trabalho de parto e parto. Rev. Rene. [Internet] 2010; 11(n.esp) [acesso em 30 dez 2015]. Disponível:

http://www.revistarene.ufc.br/revista/index.php/revista/article/view/454/pdf.

22. Borup L, Wurlitzer W, Hedegaard M, Kesmodel US, Hvdman L. Acupuncture as pain relief during delivery: a randomized controlled trial. Birth. [Internet] 2009; 36(1) [acesso em 02 fev 2016]. Disponível:

http://dx.doi.org/10.1111/j.1523-536X.2008.00290.x.

23. Pereira ALF, de Lima TRL, Schroeter MS, Gouveia MSF, do Nascimento SD. Resultados maternos e neonatais da assistência em casa de parto. Esc. Anna Nery. [Internet] 2013; 17(1) [acesso em 02 fev 2016]. Disponível: http://dx.doi.org/10.1590/S1414-81452013000100003.

24. Medeiros J, Hamad GBNZ, Costa RRO, Chaves AEP, de Medeiros SM. Métodos não farmacológicos no alívio da dor de parto: percepção de puérperas. Espaç. saúde. [Internet] 2015; 16(2) [acesso em 14 jan 2016]. Disponível: http://www.uel.br/revistas/uel/index.php/espacoparasaude/article/view/20717. 